\title{
The Epidemiology of Hypoxemic Pneumonia among Young Infants in Malawi
}

\author{
Shubhada Hooli, ${ }^{1}$ Carina King, ${ }^{2,3}$ Beatiwel Zadutsa, ${ }^{4}$ Bejoy Nambiar, ${ }^{2}$ Charles Makwenda, ${ }^{4}$ Gibson Masache, ${ }^{4}$ Norman Lufesi, ${ }^{5}$ \\ Charles Mwansambo, ${ }^{5}$ Lucas Malla, ${ }^{6}$ Anthony Costello, ${ }^{2}$ Tim Colbourn, ${ }^{2}$ and Eric D. McCollum ${ }^{7,8 *}$ \\ ${ }^{1}$ Department of Pediatrics, Section of Emergency Medicine, Baylor College of Medicine, Houston, Texas; ${ }^{2}$ Institute for Global Health, \\ University College London, London, United Kingdom; ${ }^{3}$ Department of Global Public Health, Karolinksa Institutet, Stockholm, Sweden; \\ ${ }^{4}$ Parent and Child Health Initiative, Lilongwe, Malawi; ${ }^{5}$ Republic of Malawi Ministry of Health, Lilongwe, Malawi; ${ }^{6}$ Kenya Medical Research \\ Institute-Wellcome Trust, Nairobi, Kenya; ${ }^{7}$ Division of Pulmonology, Department of Pediatrics, \\ Johns Hopkins School of Medicine, Baltimore, Maryland; ${ }^{8}$ Department of International Health, Johns Hopkins Bloomberg School \\ of Public Health, Baltimore, Maryland
}

\begin{abstract}
We describe hypoxemic pneumonia prevalence in outpatient and inpatient settings, in-hospital mortality, and clinical guideline performance for identifying hypoxemia in young infants in Malawi. In this retrospective analysis of a prospective cohort study, we investigate infants younger than 2 months participating in pneumonia surveillance at seven hospitals and 18 outpatient health centers in Malawi between 2011 and 2014. Logistic regression, multiple imputation with chained equations, and pattern mixture modeling were used to determine the association between peripheral capillary oxyhemoglobin saturation $\left(\mathrm{SpO}_{2}\right)$ levels and hospital mortality. We describe outpatient clinician hospital referral recommendations based on clinical characteristics and $\mathrm{SpO}_{2}$ distributions. Among 1,879 analyzed cases, $\mathrm{SpO}_{2}<90 \%$ was more prevalent among outpatient health center cases compared with hospitalized cases (22.6\% versus 13.5\%, 95\% Cl: 17.6-28.4\% and $12.0-15.3 \%$, respectively). A larger proportion of hospitalized infants had signs of respiratory distress compared with infants at health centers $(67.7 \%$ versus $56.6 \%, P<0.001)$ and most hospitalized infants were boys $(56.7 \%$ versus $40.6 \%, P<0.001)$. An $\mathrm{SpO}_{2}$ of $90-92 \%$ and $<90 \%$ was associated with similarly increased odds of in-hospital mortality (adjusted odds ratio [aOR]: 4.3 and 4.4, 95\% Cl: 1.7-11.1 and 1.8-10.5, respectively). Unrecorded, or unobtainable, $\mathrm{SpO}_{2}$ was highly associated with mortality ( $n=127$, aOR: 18.1; 95\% Cl: 7.6-42.8). Four of $22(18 \%)$ infants at health centers who did not meet clinical referral criteria had an $\mathrm{SpO}_{2} \leq 92 \%$. Clinicians should consider hospital referral in young infants with a $\mathrm{SpO}_{2} \leq 92 \%$. Infants with unobtainable $\mathrm{SpO}_{2}$ readings should be considered a high-risk group, and hospital referral of these cases may be appropriate.
\end{abstract}

\section{INTRODUCTION}

In 2015 lower respiratory tract infections were the leading cause of death in children younger than 5 years. ${ }^{1}$ Despite an overall reduction in pneumonia-related mortality, infants younger than 2 months continue to have high case-fatality rates. ${ }^{1}$ The 2014 WHO Integrated Management of Childhood Illness (IMCl) guidelines used in outpatient health facilities in low- and middle-income countries (LMICs) state that in children aged 2-59 months, a peripheral capillary oxyhemoglobin saturation $\left(\mathrm{SpO}_{2}\right)<90 \%$ warrants hospital referral. ${ }^{2}$ The IMCI guidelines provide no referral guidance when pulse oximetry is used in infants younger than 2 months. Studies suggest that moderate hypoxemia $\left(\mathrm{SpO}_{2} 90-92 \%\right)$ independently confers an increased risk of mortality in children aged 2-59 months. , $^{3,4}$ Most frontline facilities in LMICs do not have pulse oximeters, devices considered the standard for noninvasively measuring $\mathrm{SpO}_{2}$, ${ }^{5,6}$ and physical examination findings alone do not reliably detect hypoxemia. ${ }^{7-11}$ Previously, we reported that $68.7 \%$ of Malawian children aged 2-59 months with hypoxemia were misidentified as appropriate for outpatient care when screened using the $2014 \mathrm{WHO}$ IMCI guidelines without pulse oximetry. ${ }^{12}$

We describe the epidemiology of hypoxemia in Malawian infants younger than 2 months with pneumonia using data collected prospectively during a pneumococcal conjugate vaccine effectiveness study. ${ }^{13}$ We determined 1) hypoxemia prevalence and 2) the association between inpatient mortality

\footnotetext{
*Address correspondence to Eric D. McCollum, Eudowood Division of Pediatric Respiratory Services, Department of Pediatrics, the Johns Hopkins School of Medicine, Rubenstein Child Health Bldg., \#3150, 200 North Wolfe St., Baltimore, MD 21287. E-mail: emccoll3@ jhmi.edu
}

and different thresholds of hypoxemia, and 3) if clinical guidelines alone, without pulse oximetry, identified hypoxemic young infants requiring hospital referral.

\section{MATERIALS AND METHODS}

Setting. Malawi is a landlocked country in sub-Saharan Africa with 17.6 million people, $51.5 \%$ of whom live on less than $\$ 1.25$ per day. ${ }^{14,15}$ Community health workers, nurses, and nonphysician clinicians deliver health center services. More than $90 \%$ of hospital-based care is provided at government-operated district hospitals free of charge, and church-operated rural hospitals charge small fees and have limited inpatient capacity. ${ }^{16}$ Our study sites included 18 health centers, four rural hospitals, two district hospitals, and one regional tertiary referral hospital in Mchinji and Lilongwe districts at an altitude of 1,000-1,200 meters above sea level.

Data collection. This is a retrospective secondary analysis of a prospective cohort study evaluating pneumococcal vaccine effectiveness. Full details of training methodology and quality assessment are published elsewhere. ${ }^{12,13}$ Using procedures that had been in place and embedded within routine clinical care since 2001, we conducted active pneumonia surveillance. Government healthcare providers were trained to use pulse oximeters using a standardized protocol and collected data during routine care of children aged 0-59 months with cough and/or difficulty breathing from October 2011 to June 2014. At district and referral hospitals, lay health workers, called vital sign assistants, were trained to ensure that vital signs, including $\mathrm{SpO}_{2}$, were recorded for each patient. ${ }^{17}$

Pulse oximeters outfitted with adult clip probes (Acare Technology, Xinzhuang, Taiwan, China), validated in Malawian infants younger than 2 months, were supplied by the Lifebox Foundation (London, England). ${ }^{18}$ Providers applied 
the probe to the great toe of children weighing $<10 \mathrm{~kg}$ or aged 2 years or younger. Hospital staff completed case report forms on all patients. ${ }^{19}$ All participating healthcare providers were retrained at the study's midpoint (early 2013) by a pediatric pulmonologist and Malawi Ministry of Health $(\mathrm{MoH})$ staff. Monthly supervisory visits by study staff consisted of direct observation of patient care, including pulse oximetry use, and review of case classification, management, and data record quality. Supervisory visits emphasized case identification and remediation of poorly performing sites. Weekly death audits at district and rural hospitals were performed to ensure case report forms agreed with facility death registers. Using this same dataset, we previously published a description of the epidemiology of hypoxemia and the performance of the 2014 WHO IMCl guidelines in identifying hypoxemia among Malawian children aged 2-59 months. ${ }^{12}$

Definitions. Table 1 describes hospital referral criteria for pneumonia according to the 2000 Malawi MoH and 2014 WHO IMCI guidelines

Inclusion and exclusion criteria. In the original vaccine effectiveness study, all children aged 0-59 months with cough and/or shortness of breath met the study inclusion criteria. ${ }^{13}$ In this study, only infants younger than 2 months were included. Those who received supplemental oxygen at the time pulse oximetry was measured were excluded. Cases with documented clinical findings of pneumonia (Table 1) were included in the primary analysis except when we evaluated if clinical guidelines identify all hypoxemic infants at outpatient health centers as hospital referral eligible.

Analysis. Hypoxemia prevalence. We compared the clinical characteristics and prevalence of hypoxemia at different thresholds among eligible infants at hospitals and outpatient health centers. Data were described using Student's $t$-test for normally distributed data, Mann-Whitney U for nonparametric data, and chi-squared and Fisher's exact tests for proportions.

Association between in-hospital mortality and hypoxemia. Deaths were recorded only in the hospital dataset. If outcome data were missing, we assumed the child did not die in the hospital as we conducted weekly intensive death audits. We used multivariable logistic regression to measure the association between inpatient mortality and $\mathrm{SpO}_{2}$ at different thresholds. We defined the following as potential confounders a priori as they are parameters included in the $\mathrm{WHO} I \mathrm{MCl}$ guidelines and based on our clinical experience: gender, degree of malnutrition defined by WHO weight-for-age z-score, lower chest wall indrawing (retractions), audible wheezing, severe respiratory distress (grunting, head nodding, or nasal flaring), tachypnea (respiratory rate $\geq 60,70$, or 80 breaths per minute), tachycardia (heart rate $\geq 160,180$, or 190 beats per minute), bradycardia (heart rate $<110$ or 100 beats

TABLE 1

Hospital referral criteria for infants aged 0-2 months with pneumonia

\begin{tabular}{cc}
\hline 2000 Malawi Ministry of Health & $\begin{array}{c}2014 \text { WHO Integrated Management } \\
\text { of Childhood lllness }\end{array}$ \\
\hline $\begin{array}{l}\text { Fast breathing }{ }^{*} \text { or chest wall } \\
\text { indrawing or danger }\end{array}$ & $\begin{array}{c}\text { Fast breathing }{ }^{*} \text { or chest wall indrawing } \\
\text { or poor feeding or convulsions or } \\
\text { signs } \dagger\end{array}$ \\
$\begin{array}{l}\text { temperature } \geq 37.5^{\circ} \mathrm{C} \text { or }<35.5^{\circ} \mathrm{C} \text { or } \\
\text { decreased movement }\end{array}$
\end{tabular}

${ }^{*}$ Fast breathing defined as a respiratory rate $>60$ breaths per minute.

† Danger signs include central cyanosis, severe respiratory distress (grunting, head nodding, and nasal flaring), stridor in a calm child, a general WHO danger sign (inability to drink and/or breastfeed, lethargy or unconscious, or convulsions), and apnea. per minute), fever (temperature $\geq 37.5^{\circ} \mathrm{C}$, as per the WHO guidelines), hypothermia (temperature $<35.5^{\circ} \mathrm{C}$ ), and general WHO danger signs (central cyanosis, stridor, inability to drink, lethargy, or convulsions). ${ }^{20}$ We chose tachypnea, tachycardia, and bradycardia cutoffs based on population normative values and international guidelines published by the American Heart Association Pediatric Advanced Life Support program and the WHO. ${ }^{20}$ All potential confounders were investigated in bivariate analysis, and those with a $P$-value $<0.2$ were included in the final model. In the primary analysis (Model 1), missing values for confounders were imputed, using multiple (10) imputations with chained equations (MICEs), and unrecorded $\mathrm{SpO}_{2}$ was included as a nominal group. ${ }^{21}$

We observed staff unsuccessfully attempt to obtain pulse oximetry measurements, with $\mathrm{SpO}_{2}$ left unrecorded in these cases, suggesting these data may be missing not at random (MNAR). To test our assumption, we conducted three sensitivity analyses. In Model 2, we created a composite binary pattern mixture variable to identify cases that had a high degree of missing confounders. ${ }^{22}$ First, we compared missing confounders by survival outcomes. Peripheral oxyhemoglobin saturation was excluded from consideration, as our goal was to determine if pulse oximetry readings were MNAR. Tachycardia and bradycardia were excluded, given that $\mathrm{SpO}_{2}$ and heart rates were both measured using pulse oximetry. Confounders were included in the composite binary pattern mixture variable if they were missing in more than $25 \%$ of the deceased or surviving cases. Cases were identified as having high instance of missing confounders if they were missing one of the identified confounders. We treated unrecorded $\mathrm{SpO}_{2}$ as a nominal group and used MICE to account for missing confounders but included a binary pattern mixture variable in the multivariable logistic regression model. In Model 3, unrecorded $\mathrm{SpO}_{2}$ and missing confounders were imputed using MICE. Model 4 was the same as Model 3 but included the pattern mixture variable.

Referral guidelines and pulse oximetry. We evaluated the added value of using pulse oximetry among young infants at outpatient health centers in two ways. First, we determined the proportion of cases classified as appropriate for outpatient management based on Malawi MoH clinical guidelines alone (Table 1). Second, we retrospectively applied the 2014 WHO $\mathrm{IMCl}$ guidelines to compare the number of infants meeting the referral criteria, assuming perfect application of these guidelines, with and without the availability of pulse oximetry.

Stata software version 14.2. (StataCorp. 2015, College Station, TX) was used to perform all analyses.

Ethical approval. The National Health Sciences Research Committee of Malawi (protocol: 941) and the Ethics Committee of University College London (protocol: 2006/002) provided ethical approval. Given that the study collected routine clinical data, the ethical boards did not require informed consent.

\section{RESULTS}

A total of 2,071 encounters were evaluated for study inclusion. Documented clinical findings warranting either referral or hospitalization as per the Malawi MoH guidelines were noted in 235 (90.0\%) encounters at health centers and 1,694 $(93.6 \%)$ encounters at hospitals. Of the hospitalized patients, $76(4.5 \%)$ were excluded, leaving 1,618 hospitalized cases in the analysis (Figure 1). 


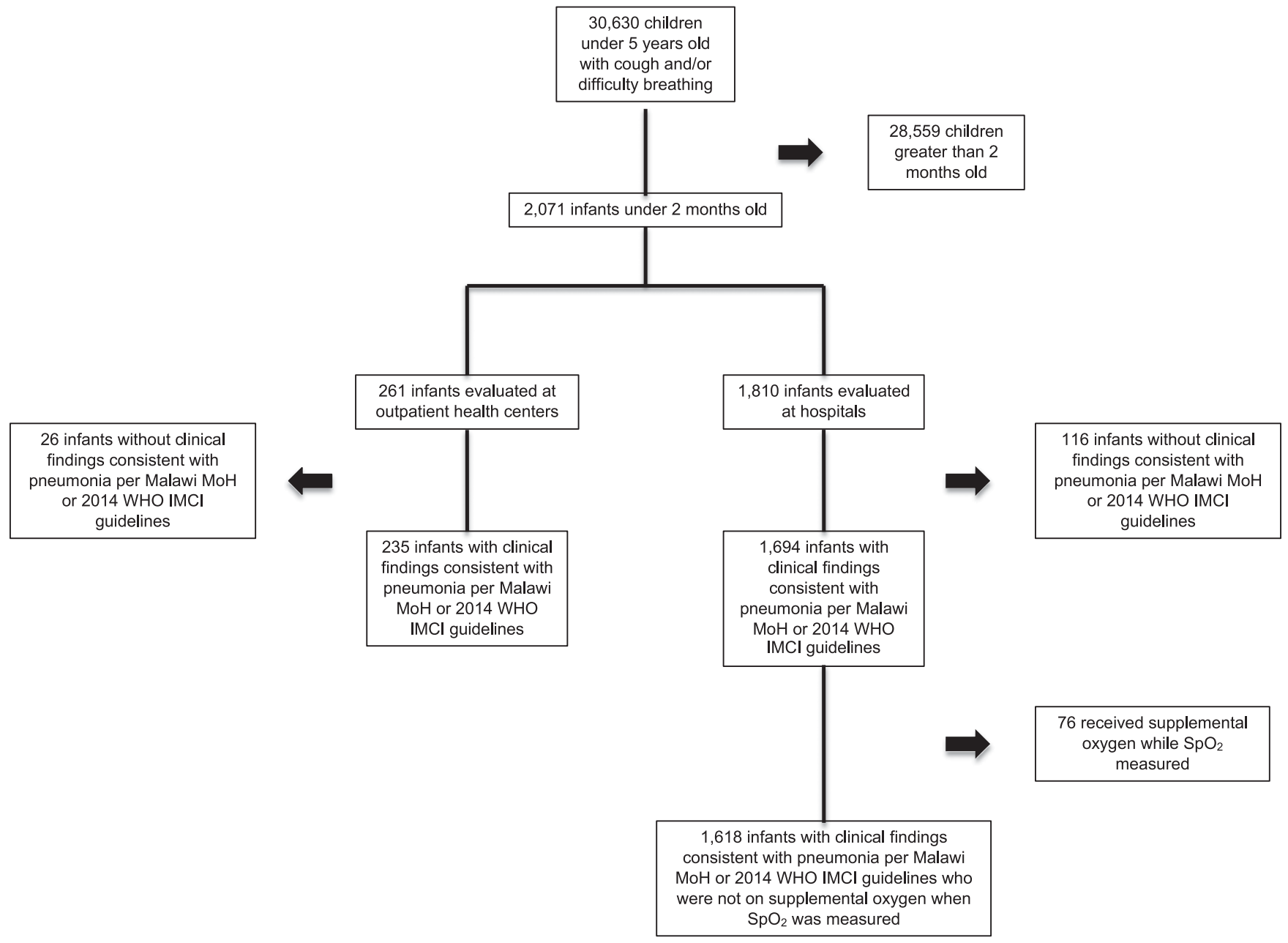

FIGURE 1. Cases included in analysis.

Hypoxemia prevalence. A larger proportion of cases at outpatient health centers, compared with hospitals, had an $\mathrm{SpO}_{2}<90 \%$ or had an unrecorded $\mathrm{SpO}_{2}$. Hospitalized infants, compared with those evaluated at health centers, had a higher prevalence of chest wall indrawing and severe respiratory distress. A larger proportion of hospitalized infants were male versus female $(56.7 \%$ versus $40.6 \%, P<0.001)$ (Table 2 ).

At outpatient health centers, infants with an unrecorded $\mathrm{SpO}_{2}$ had a lower mean weight (3.9 versus $4.6 \mathrm{~kg}, P=0.03$ ) and a higher prevalence of $\mathrm{WHO}$ danger signs (44.8\% versus $24.3 \%, P=0.02$ ) compared with those with a recorded $\mathrm{SpO}_{2}$ (Table 3). A larger proportion of hospitalized infants with an unrecorded $\mathrm{SpO}_{2}$ measurement, compared with hospitalized infants with a recorded $\mathrm{SpO}_{2}$, had $\mathrm{WHO}$ danger signs $(33.1 \%$ versus $22.5 \%, P<0.001$ ), and a smaller proportion had fever (26.0\% versus $32.7 \%, P=0.02)$. Otherwise, clinical characteristics of patients with recorded versus unrecorded $\mathrm{SpO}_{2}$ measurements were not statistically different. Notably, a large proportion of cases with unrecorded $\mathrm{SpO}_{2}$ measurements were missing other covariates (Table 3).

Association between in-hospital mortality and hypoxemia. The overall hospital case fatality rate was $3.6 \%$ $(n=59)$. Generally, death cases had a higher prevalence of missing data (Supplemental Material 1). Table 4 describes the bivariate analysis for confounder selection and the adjusted odds (aOR) for mortality using logistic regression with MICE and pattern mixture modeling. In Model 1, infants with an $\mathrm{SpO}_{2}$ 90-92\% or an $\mathrm{SpO}_{2}<90 \%$ had a similarly increased aOR for in-hospital mortality at $4.3(95 \% \mathrm{Cl}: 1.7-11.1)$ and $4.4(95 \% \mathrm{Cl}$ : 1.8-10.5), respectively. Children with an unrecorded $\mathrm{SpO}_{2}$ had the highest case fatality rate at $18.9 \%(95 \% \mathrm{Cl}$ : 13.0-26.7\%) and an aOR for in-hospital mortality of 18.1 (95\% Cl: 7.6, 42.8). Model 2, using a binary pattern mixture composite variable for missing danger signs or malnutrition, produced similar results to Model 1, noting the wide Cls for each. Models 3 and 4, imputing unrecorded $\mathrm{SpO}_{2}$ using MICE, produced aOR for mortality associated with an $\mathrm{SpO}_{2} 90-92 \%$ and an $\mathrm{SpO}_{2}<90 \%$ similar to those of Models 1 and 2. There was a modest increase in the associated odds for mortality attributed to the pattern mixture variable in Model 4, compared with Model 2, at 3.7 versus 2.4 (aOR 95\% Cl: 1.2, 4.7 and 1.9, 7.2 , respectively).

Referral guidelines and pulse oximetry. At outpatient health centers, a similar proportion of infants were eligible for hospital referral based on the Malawi MoH $(n=235 / 261)$ and $2014 \mathrm{WHO}$ IMCI $(n=237 / 261)$ guidelines when retrospectively assuming pulse oximetry was unavailable $(90.0 \%$ versus $90.8 \%, P=0.88$ ). Four cases had too much missing data to 
TABLE 2

Observed clinical characteristics of infants admitted to hospitals or evaluated at health centers diagnosed with pneumonia based on the 2000 Malawi Ministry of Health guidelines

\begin{tabular}{|c|c|c|c|}
\hline Patient and clinical characteristics & Hospitals, $N=1,618$ & Health centers, $N=235$ & $P$-value \\
\hline $\mathrm{SpO}_{2}$, median value, \% (IQR) & $96(92-98)$ & 94 (89-97) & $<0.001$ \\
\hline $\mathrm{SpO}_{2}>92 \%, n(\%, 95 \% \mathrm{Cl})$ & $1,088(67.2,64.9-69.5)$ & $123(52.3,45.9-58.7)$ & $<0.001$ \\
\hline $\mathrm{SpO}_{2} 90-92 \%, n(\%, 95 \% \mathrm{Cl})$ & $184(11.4,9.9-13.0)$ & $30(12.8,9.1-17.7)$ & \\
\hline $\mathrm{SpO}_{2}<90 \%, n(\%, 95 \% \mathrm{Cl})$ & $219(13.5,12.0-15.3)$ & $53(22.6,17.6-28.4)$ & \\
\hline Unrecorded $\mathrm{SpO}_{2}, n(\%, 95 \% \mathrm{Cl})$ & $127(7.9,6.6-9.3)$ & $29(12.3,8.7-17.2)$ & \\
\hline Respiratory rate, median (IQR) & $64(59-69)$ & $63(61-67)$ & 0.50 \\
\hline Missing, $n(\%)$ & $110(6.8)$ & $16(6.8)$ & \\
\hline Weight (kg), mean (SD) & $4.4(1.6)$ & $4.5(1.5)$ & 0.23 \\
\hline Missing, $n(\%)$ & $69(4.3)$ & $12(5.1)$ & \\
\hline Male, $n(\%, 95 \% \mathrm{Cl})$ & $918(56.7,54.3-59.1)$ & $113(48.1,41.7-54.5)$ & 0.07 \\
\hline Female, $n(\%, 95 \% \mathrm{Cl})$ & $657(40.6,38.2-43.0)$ & $105(44.7,38.4-51.1)$ & \\
\hline Missing, $n(\%)$ & $43(2.7)$ & $17(7.2)$ & \\
\hline WAZ > $-2, n(\%, 95 \% \mathrm{Cl})$ & $1,256(77.6,75.5-79.6)$ & $175(74.5,68.5-79.6)$ & 0.37 \\
\hline$-3<$ WAZ $\leq-2, n(\%, 95 \% \mathrm{Cl})$ & $172(10.6,9.2-12.2)$ & $26(11.1,7.6-15.8)$ & \\
\hline WAZ $\leq-3, n(\%, 95 \% \mathrm{Cl})$ & $85(5.3,4.3-6.5)$ & $7(3.0,1.4-6.1)$ & \\
\hline Missing, $n(\%)$ & $105(6.5)$ & $27(11.5)$ & \\
\hline Chest wall indrawing, $n(\%, 95 \% \mathrm{Cl})$ & $1,471(90.9,89.4-92.2)$ & $165(70.2,64.0-75.7)$ & $<0.001$ \\
\hline Missing, $n(\%)$ & $67(4.1)$ & $7(3.0)$ & \\
\hline WHO danger signs, ${ }^{*} n(\%, 95 \% \mathrm{Cl})$ & $377(23.3,21.3-25.4)$ & $63(26.8,21.5-32.9)$ & 0.64 \\
\hline Missing, $n(\%)$ & $196(12.1)$ & $10(4.3)$ & \\
\hline Severe respiratory distress, $\uparrow n(\%, 95 \% \mathrm{Cl})$ & $1,096(67.7,65.4-70.0)$ & $133(56.6,50.2-62.8)$ & $<0.001$ \\
\hline Missing, $n(\%)$ & $147(9.1)$ & $8(3.4)$ & \\
\hline
\end{tabular}

$\mathrm{IQR}=$ interquartile range; $\mathrm{SpO}_{2}=$ peripheral capillary oxyhemoglobin saturation; WAZ = WHO weight-for-age $z$-score.

* Danger signs include central cyanosis, stridor in a calm child, apnea, or a general danger sign (inability to drink and/or breastfeed, lethargy or unconscious, or convulsions).

† Severe respiratory distress: grunting, head nodding, or nasal flaring.

determine if they met the Malawi MoH referral guidelines and were excluded from the rest of the analyses. The Malawi MoH clinical guidelines, without pulse oximetry, classified 22/257 (8.6\%) infants as appropriate for outpatient management. One $(5 \%)$ of these 22 infants had an $\mathrm{SpO}_{2}<90 \%$, three (14\%) had an $\mathrm{SpO}_{2}$ of $90-92 \%$, and pulse oximetry was unrecorded for three (14\%) (Figure 2). Application of the Malawi MoH guidelines without pulse oximetry misclassified $2 \%(1 / 54)$ with an $\mathrm{SpO}_{2}<90 \%$ and $5 \%$ (4/87) of infants with an $\mathrm{SpO}_{2} \leq 92 \%$ as appropriate for outpatient management (Supplemental

TABLE 3

Clinical characteristics of infants with pneumonia categorized by successful pulse oximetry measurement and clinical setting

\begin{tabular}{|c|c|c|c|c|c|c|}
\hline \multirow[b]{2}{*}{ Variable } & \multicolumn{3}{|c|}{ Hospitals $(n=1,618)$} & \multicolumn{3}{|c|}{ Health centers $(n=235)$} \\
\hline & Successful, $n(\%)$ & Unsuccessful, $n(\%)$ & $P$ & Successful, $n(\%)$ & Unsuccessful, $n$ (\%) & $P$ \\
\hline $\begin{array}{l}\text { Peripheral capillary } \\
\text { oxyhemoglobin } \\
\text { saturation measurement }\end{array}$ & $1,491(92.2)$ & $127(7.8)$ & - & $206(87.7)$ & $29(12.3)$ & - \\
\hline Male & $855(57.3,54.8-59.8)$ & $63(49.6,41.0-58.3)$ & 0.31 & $103(50.0,43.2-56.8)$ & $10(34.5,19.4-53.5)$ & 0.07 \\
\hline Female & $603(40.4,38.0-43.0)$ & $54(42.5,34.2-51.3)$ & & $87(42.2,35.6-49.1)$ & $18(62.1,43.2-77.9)$ & \\
\hline Missing & $33(2.2)$ & $10(7.9)$ & & $16(7.8)$ & $1(3.5)$ & \\
\hline Weight in kg, mean (SD) & $4.4(1.7)$ & $4.3(1.3)$ & 0.80 & $4.6(1.5)$ & $3.9(0.9)$ & 0.03 \\
\hline Missing & $33(2.2)$ & $36(28.4)$ & & $6(2.9)$ & $6(20.7)$ & \\
\hline $\begin{array}{l}\text { Respiratory rate, median } \\
\text { (interquartile range) }\end{array}$ & $64(59-68)$ & $65(59-71)$ & 0.61 & $63(60-67)$ & $64(62-67)$ & 0.65 \\
\hline Missing & $39(2.6)$ & $71(55.9)$ & & $8(3.9)$ & $8(27.6)$ & \\
\hline Heart rate $\geq 190$ & $89(6.0,4.9-7.3)$ & 0 & 0.16 & $2(1.0,0.2-3.8)$ & 0 & 0.66 \\
\hline Heart rate $<110$ & $67(4.5,3.6-5.7)$ & $2(1.6,0.4-6.1)$ & & $96(46.6,39.8-53.5)$ & $1(3.5,0.5-21.6)$ & \\
\hline Missing & $24(1.6)$ & $113(89.0)$ & & $1(0.5)$ & $25(86.2)$ & \\
\hline Fever & $488(32.7,30.4-35.2)$ & $33(26.0,19.1-34.3)$ & 0.02 & $84(40.8,34.2-47.7)$ & $9(31.3,16.8-50.2)$ & 0.29 \\
\hline Hypothermia & $29(2.0,1.4-2.8)$ & $4(3.2,1.2-8.1)$ & & $5(2.4,1.0-5.7)$ & $2(6.9,1.7-24.4)$ & \\
\hline Missing & $26(1.7)$ & $50(39.4)$ & & $12(5.8)$ & $6(20.7)$ & \\
\hline WHO danger signs* & $335(22.5,20.4-24.7)$ & $42(33.1,25.4-41.7)$ & $<0.001$ & $50(24.3,18.9-30.6)$ & $13(44.8,27.8-63.2)$ & 0.02 \\
\hline Missing & $163(10.9)$ & $33(26.0)$ & & $9(4.4)$ & $1(3.5)$ & \\
\hline Severe respiratory distress $\dagger$ & $1,015(68.1,65.7-70.4)$ & $81(63.8,55.0-71.7)$ & 0.68 & $113(54.9,48.0-61.6)$ & $20(69.0,49.8-83.2)$ & 0.23 \\
\hline Missing & $117(7.8)$ & $20(15.8)$ & & $8(3.9)$ & 0 & \\
\hline Chest wall indrawing & $1,349(90.5,88.9-91.9)$ & $122(96.1,90.9-98.4)$ & 0.40 & $142(68.9,62.2-74.9)$ & $23(79.3,60.5-90.6)$ & 0.37 \\
\hline Missing & $66(4.4)$ & $1(0.8)$ & & $7(3.4)$ & 0 & \\
\hline $\begin{array}{l}2014 \text { integrated management } \\
\text { of childhood illness } \\
\text { referral eligible } \neq\end{array}$ & $1,486(99.7)$ & $127(100.0)$ & 1.00 & 203 (98.5) & $28(96.6)$ & 0.77 \\
\hline Missing & $5(0.3)$ & 0 & & $3(1.5)$ & $1(3.5)$ & \\
\hline
\end{tabular}




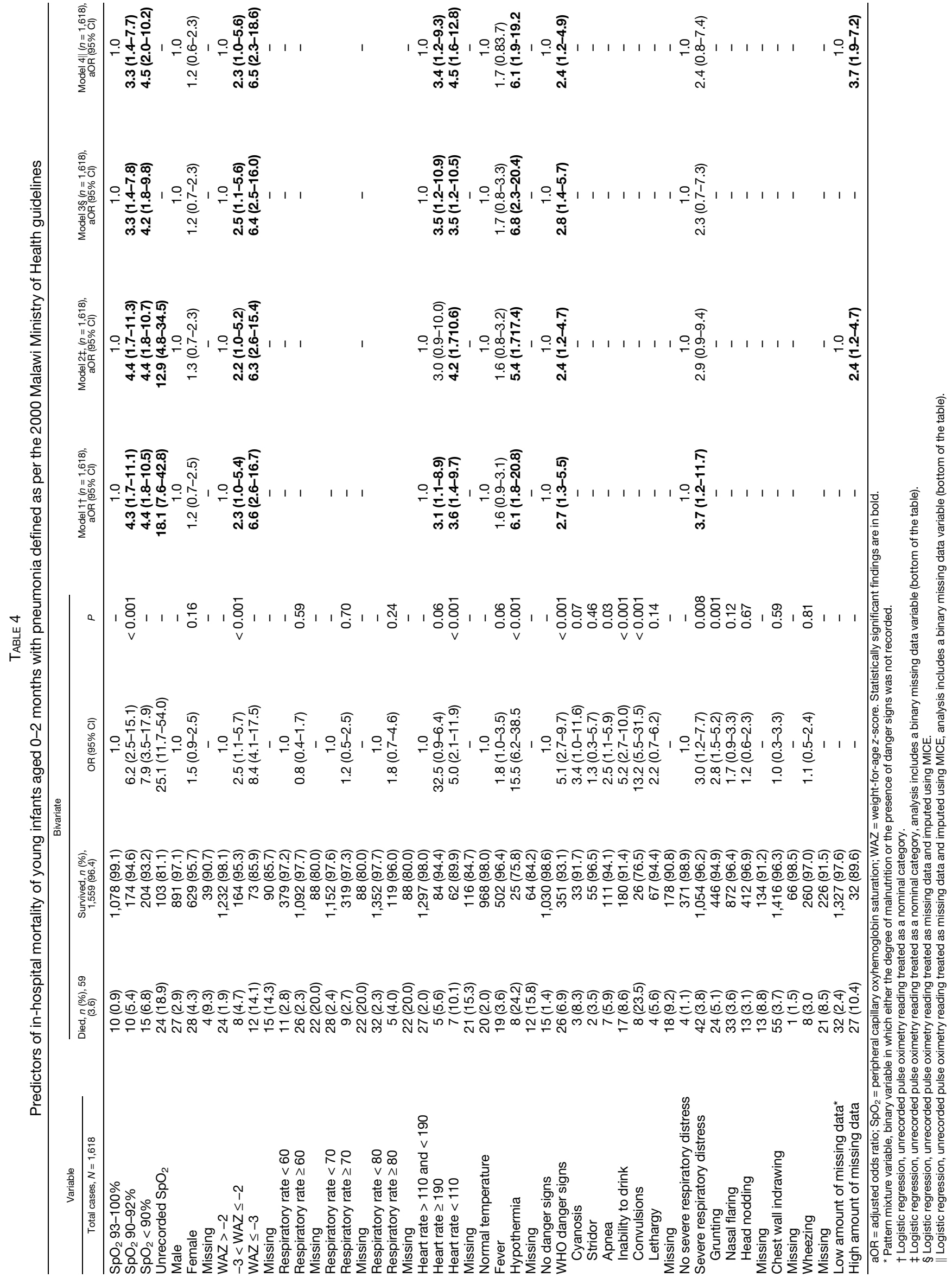



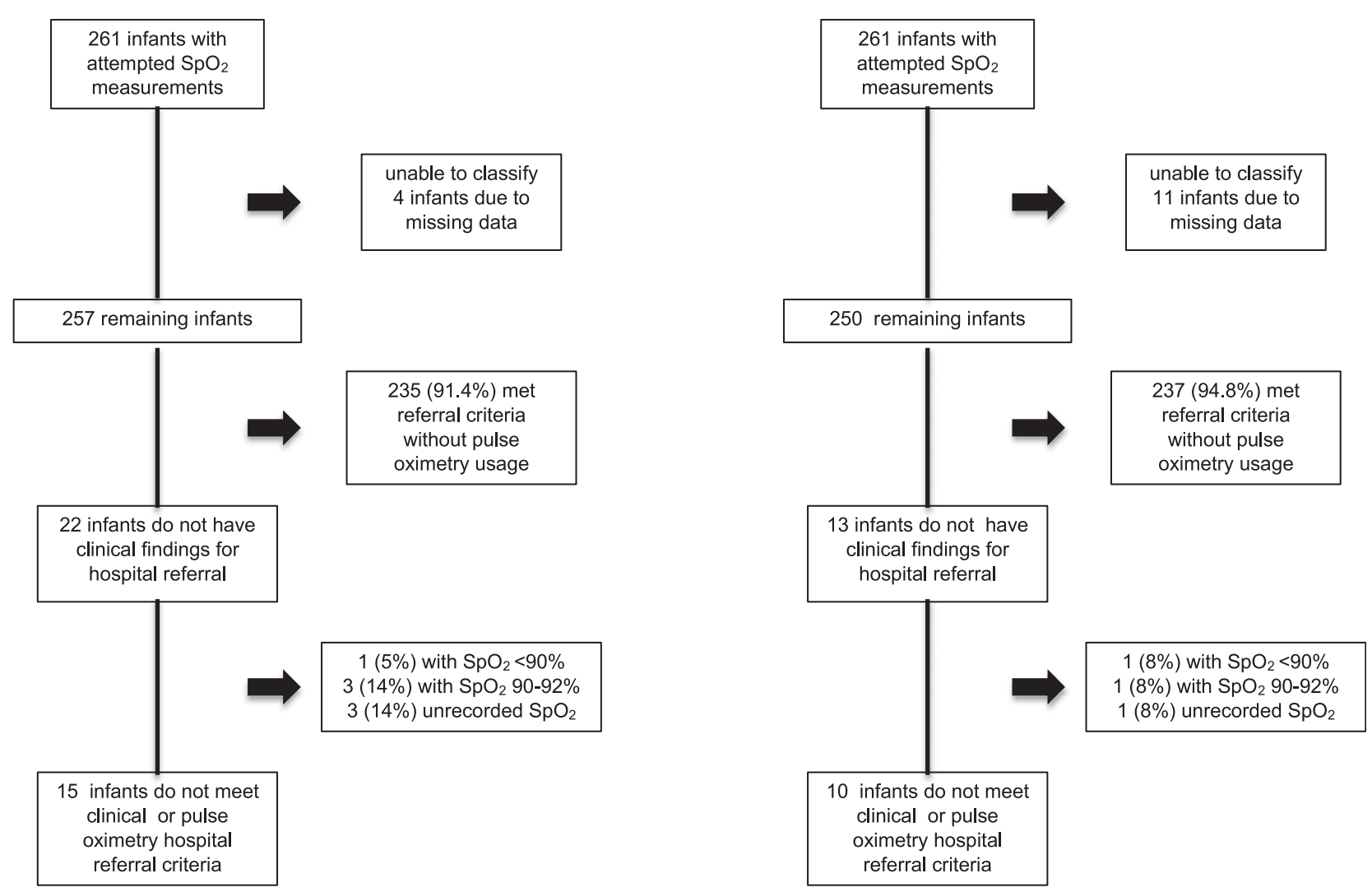

FIGURE 2. Performance of the Malawi Ministry of Health and 2014 WHO Integrated Management of Childhood Illness guidelines at identifying hypoxemic infants with cough and/or shortness of breath.

Material 2). Comparatively, the $2014 \mathrm{WHO} \mathrm{IMCl}$ guidelines misclassified $2 \%(1 / 54)$ with an $\mathrm{SpO}_{2}<90 \%$ and $1 / 87(1 \%)$ of infants with an $\mathrm{SpO}_{2} \leq 92 \%$ (Supplemental Material 3). Of the 13 infants who did not meet hospital referral clinical criteria, one (8\%) had an $\mathrm{SpO}_{2}<90 \%$ and one (8\%) had an $\mathrm{SpO}_{2} \leq 92 \%$.

\section{DISCUSSION}

We analyzed 1,879 cases of Malawian infants younger than 2 months presenting to outpatient health centers and hospitals with WHO-defined pneumonia and found that infants with an $\mathrm{SpO}_{2}$ of $90-92 \%$ had a near-equal aOR for inpatient mortality compared with those with an $\mathrm{SpO}_{2}<90 \%$. This is not surprising as $\mathrm{SpO}_{2}$ in some cases may overestimate arterial blood oxygen saturation $\left(\mathrm{SaO}_{2}\right) \cdot{ }^{23} \mathrm{An} \mathrm{SpO}_{2}$ of $89-92 \%$ on average correlates with an $\mathrm{SaO}_{2}$ of $90 \%$ and partial pressure of oxygen of $60 \mathrm{mmHg}$ in an otherwise healthy child. ${ }^{24}$ The 2013 WHO Pocket Book of Hospital Care, used in hospital settings, recommends starting supplemental oxygen in young infants with an $\mathrm{SpO}_{2}<90 \%$ or those with central cyanosis or severe respiratory distress. ${ }^{25}$ We found a high (24.9-35.4\%) prevalence of clinically meaningful hypoxemia $\left(\mathrm{SpO}_{2} \leq 92 \%\right)$ similar to other studies that defined hypoxemia as an $\mathrm{SpO}_{2}<$ 90\%. ${ }^{26-28}$ Among the small proportion of infants with upper respiratory tract infection symptoms who did not meet the 2014 WHO IMCl hospital referral criteria, 15\% were still hypoxemic $\left(\mathrm{SpO}_{2} \leq 92 \%\right)$ and, therefore, at high risk of mortality. The $\mathrm{WHO} I \mathrm{MCl}$ guidelines, used by health workers at outpatient health centers, do not provide any referral guidance when $\mathrm{SpO}_{2}$ is measured in infants younger than 2 months but state that older children aged 2-59 months with an $\mathrm{SpO}_{2}<$ $90 \%$, independent of other examination findings, should be referred to hospitals. ${ }^{2}$ Without more specific guidance from the $\mathrm{WHO}$ on the application of $\mathrm{SpO}_{2}$ measurements among young infants, clinicians may or may not apply these recommendations to infants younger than 2 months. Our findings generate uncertainty around the generalizability of the current WHO guidelines that lack pulse oximetry recommendations for young infants in Malawi and potentially other LMICs.

Notably, we found a markedly elevated aOR of mortality associated with an unrecorded $\mathrm{SpO}_{2}$ which was robust to multiple modeling approaches. These infants comprised $8 \%$ of hospitalized cases and accounted for $41 \%$ of all deaths. Both health center outpatients and hospital inpatients had a higher prevalence of WHO danger signs among infants with an unrecorded $\mathrm{SpO}_{2}$. One explanation for the associated increased mortality, based on direct observations, is that $\mathrm{SpO}_{2}$ was not documented after attempted but failed measurements. A high proportion of these cases were likely infants with circulatory compromise due to shock and poor peripheral perfusion that rendered obtaining pulse oximetry readings difficult. Indicators of poor perfusion such as capillary refill 
time, cool extremities, or history of reduced frequency of urination, which are included in the WHO's Emergency Triage and Treatment guidelines, ${ }^{29}$ are not included in the $\mathrm{WHO} \mathrm{IMCl}$ guidelines. A study conducted in the United States found that the capillary refill time and cool extremities had moderate to poor inter-rater reliability. ${ }^{30}$ We recommend further exploration on the feasibility and reliability of healthcare providers collecting these signs, especially in the context of the inability to obtain $\mathrm{SpO}_{2}$ measurement.

Like many LMICs, pulse oximetry and supplemental oxygen availability and uptake are poor in Malawi, especially in outpatient health centers. ${ }^{5,6,31}$ This situation is particularly disturbing, as the prevalence of hypoxemia, regardless of the threshold used, was higher among outpatients. A retrospective analysis of the Malawi Child Lung Health Program from 2001 to 2012 found that among infants younger than 2 months, referral from a health center was an independent mortality risk factor. ${ }^{16}$ This finding may reflect discrepancies among care-seeking behaviors of communities living near hospitals, challenges in the referral pathway, or lack of oxygen and antibiotic availability at health centers. Some of these children may have died en route to the hospital or families may not go to the hospital despite referral. The use of pulse oximetry in outpatient settings has the potential to strengthen multiple aspects of young infant primary care, including improving the identification of true high-risk cases, strengthen referral recommendations by healthcare providers currently relying solely on nonspecific clinical signs such as respiratory rate, increase the uptake of referral recommendations by families, and ultimately improve clinical care through refinement of hospital transfer processes and supplemental oxygen prescribing practices. Further research will be needed to more fully evaluate pulse oximetry effects on the primary care health system, especially as it pertains to young infants.

A large proportion of infants evaluated at health centers met the hospital referral criteria, even without pulse oximetry usage because of the high prevalence of tachypnea. Young infants are at high risk of rapid decompensation from serious bacterial infections including pneumonia. Given the relative high prevalence of serious bacterial infections in this age-group, the WHO guidelines set a very low threshold for hospital referral for expert evaluation or, if indicated, inpatient admission. The Malawi MoH clinical guidelines misclassified $5 \%$ of infants with cough and/or shortness of breath with an $\mathrm{SpO}_{2} \leq 92 \%$ as appropriate for outpatient management. Implementation of the $2014 \mathrm{WHO}$ IMCI guidelines would reduce this proportion by half. If healthcare workers were to adhere to the IMCl guidelines, very few infants with an $\mathrm{SpO}_{2} \leq 92 \%$ would be discharged home. This finding is in contrast with our previous finding among Malawian children aged 2-59 months that $68.7 \%$ with an $\mathrm{SpO}_{2}<90 \%$ were misclassified by the clinical guidelines as appropriate for outpatient care. $^{12}$ Our dataset does not explicitly indicate how healthcare workers may have been influenced by either a "normal" or "abnormal" $\mathrm{SpO}_{2}$, and it remains unclear whether busy healthcare workers may over-rely on $\mathrm{SpO}_{2}$ reading, compared with the clinical criteria, when making referral decisions. Further research on whether "normal" $\mathrm{SpO}_{2}$ measurements among children otherwise clinically eligible for referral may detrimentally affect referral decision-making.

Limitations. Understanding practices and outcomes in routine clinical care is essential to informing guideline and program development. Although a primary strength of our study is that these data reflect real-life clinical practices in
Malawi, programmatic data have some limitations, most notably missing data. Death cases had a high degree of missing data on other variables, limiting the certainty of our conclusions. It is possible that the need to stabilize these critically ill patients precluded hospital staff's recording of clinical data at the time of admission. We found evidence that many of these measurements were MNAR, as inclusion of the pattern mixture variable in the logistic regression model still resulted in an unrecorded $\mathrm{SpO}_{2}$ associated with an increased aOR for inhospital mortality. We did not conduct home-based follow-up to assess if families followed referral recommendations, and infants were later taken to higher level health facilities or returned for re-evaluation, developed more severe disease, or died. Hemoglobin and malaria testing were not routinely documented as part of the pneumonia surveillance program. Both would be useful in assessing illness etiology and the former to determine inherently low oxygen-carrying capacity. Finally, our conclusions regarding the proportion of hypoxemic infants with upper respiratory infection symptoms missed by clinical guidelines alone are limited by the small sample size and should be interpreted within this context.

\section{CONCLUSION}

This observational study reflects real-world pneumonia care practices for young infants at outpatient health centers and hospitals by healthcare providers in a nonclinical trial setting in Malawi. Our findings pose several important questions for further research and on areas of focus for guideline revisions with regard to use of pulse oximetry and its feasibility of implementation and operationalization. Given that this was an observational study, with missing data, caution should be taken in the interpretation of these results. Nonetheless, our findings remain compelling, particularly when considering the strong association of an $\mathrm{SpO}_{2}$ of $90-92 \%$ and $<90 \%$ with hospital mortality, along with the high prevalence of hypoxemia in outpatient settings. Importantly, our findings, if replicated elsewhere, provide evidence supporting revision of the WHO guidelines to recommend hospital referral of young infants at outpatient centers with an $\mathrm{SpO}_{2} \leq 92 \%$, or for whom a pulse oximetry reading is unobtainable.

Received July 10, 2019. Accepted for publication November 17, 2019. Published online January 20, 2020.

Note: Supplemental materials appear at www.ajtmh.org.

Acknowledgments: We thank Rashid Deula for leading efforts in data collection and curation of the original study. We thank the Republic of Malawi Ministry of Health and the communities and traditional authorities of Lilongwe and Mchinji districts of Malawi. This work is the direct result of the dedication and hard work of our field and data collection staff. We also thank the editorial assistance by B. Lee Ligon and internal review by Sue Torrey, Andrea Cruz, and Manish Shah of the Department of Pediatrics, Baylor College of Medicine, Houston, Texas. A previous version of this work was presented at the 2018 Consortium of Universities for Global Health meeting in New York, New York.

Financial support: The Bill \& Melinda Gates Foundation funded all stages of this study. E. D. M. received support from the Fogarty International Center of the National Institutes of Health (K01TW009988).

Disclaimer: A previous version of this work was presented at the 2018 Consortium of Universities for Global Health meeting in New York.

Authors' addresses: Shubhada Hooli, Department of Pediatrics, Section of Emergency Medicine, Baylor College of Medicine, 
Houston, TX, E-mail: hooli@bcm.edu. Carina King, Institute for Global Health, University College London, London, United Kingdom, and Department of Public Health Sciences, Karolinska Institutet, Stockholm, Sweden, E-mail: c.king@ucl.ac.uk. Beatiwel Zadutsa, Charles Makwenda, and Gibson Masache, Parent and Child Health Initiative, Lilongwe, Malawi, E-mails: beatzadutsa@gmail.com, charlesvmakwenda@gmail.com, and gibsonmasache2012@yahoo.com. Bejoy Nambiar, Anthony Costello, and Tim Colbourn, Institute for Global Health, University College London, London, United Kingdom, E-mails: b.nambiar@ucl.ac.uk, cihdcostello@ gmail.com, and t.colbourn@ucl.ac.uk. Norman Lufesi and Charles Mwansambo, Republic of Malawi Ministry of Health, Lilongwe, Malawi, E-mails: nlufesi@gmail.com and cmwansambo@gmail.com. Lucas Malla, Kenya Medical Research Institute-Wellcome Trust, Nairobi, Kenya, E-mail: Imalla@kemri-wellcome.org. Eric D. McCollum, Division of Pulmonology, Department of Pediatrics, Johns Hopkins School of Medicine, Baltimore, MD, and Department of International Health, Johns Hopkins Bloomberg School of Public Health, Baltimore, MD, E-mail: emccoll3@jhmi.edu.

\section{REFERENCES}

1. Liu L, Oza S, Hogan D, Chu Y, Perin J, Zhu J, Lawn JE, Cousens S, Mathers C, Black RE, 2016. Global, regional, and national causes of under-5 mortality in 2000-15: an updated systematic analysis with implications for the sustainable development goals. Lancet Lond Engl 388: 3027-3035.

2. World Health Organization, 2014. IMCl Chart Booklet. Available at: http://www.who.int/maternal_child_adolescent/documents/ IMCI_chartbooklet/en/. Accessed December 15, 2017.

3. Lazzerini M, Sonego M, Pellegrin MC, 2015. Hypoxaemia as a mortality risk factor in acute lower respiratory infections in children in low and middle-income countries: systematic review and meta-analysis. PLoS One 10: e0136166.

4. Hooli S, Colbourn T, Lufesi N, Costello A, Nambiar B, Thammasitboon S, Makwenda C, Mwansambo C, McCollum ED, King C, 2016. Predicting hospitalised paediatric pneumonia mortality risk: an external validation of RISC and $\mathrm{mRISC}$, and local tool development (RISC-Malawi) from Malawi. PLoS One 11: e0168126.

5. Ginsburg AS, Van Cleve WC, Thompson MI, English M, 2012. Oxygen and pulse oximetry in childhood pneumonia: a survey of healthcare providers in resource-limited settings. $J$ Trop Pediatr 58: 389-393.

6. English $\mathrm{M}$ et al., 2014. Adoption of recommended practices and basic technologies in a low-income setting. Arch Dis Child 99: 452-456.

7. Bassat $Q$ et al., 2011. Distinguishing malaria from severe pneumonia among hospitalized children who fulfilled integrated management of childhood illness criteria for both diseases: a hospital-based study in Mozambique. Am J Trop Med Hyg 85: 626-634.

8. Mwaniki MK, Nokes DJ, Ignas J, Munywoki P, Ngama M, Newton CR, Maitland K, Berkley JA, 2009. Emergency triage assessment for hypoxaemia in neonates and young children in a Kenyan hospital: an observational study. Bull World Health Organ 87: 263-270.

9. Duke T, Mgone J, Frank D, 2001. Hypoxaemia in Children with Severe Pneumonia in Papua New Guinea [Oxygen Therapy in Children]. Available at: https://www.ingentaconnect.com/content/ iuatld/ijtld/2001/00000005/00000006/art00004\#. Accessed November 19, 2018.

10. Usen S, Weber M, 2001. Clinical signs of hypoxaemia in children with acute lower respiratory infection: indicators of oxygen therapy [oxygen therapy in children]. Int $J$ Tuberc Lung Dis 5: 505-510.

11. Rao YK, Midha T, Kumar P, Tripathi VN, Rai OP, 2012. Clinical predictors of hypoxemia in Indian children with acute respiratory tract infection presenting to pediatric emergency department. World J Pediatr 8: 247-251.
12. McCollum ED et al., 2016. Pulse oximetry for children with pneumonia treated as outpatients in rural Malawi. Bull World Health Organ 94: 893-902.

13. McCollum ED et al., 2017. Impact of the 13-valent pneumococcal conjugate vaccine on clinical and hypoxemic childhood pneumonia over three years in Central Malawi: an observational study. PLoS One 12: e0168209.

14. National Statistical Office, 2018. 2018 Malawi Population and Housing Census Preliminary Report. Zomba: National Statistical Office.

15. The World Bank, 2018. Malawi Data. Available at: https:// data.worldbank.org/country/malawi. Accessed February 14, 2019.

16. Lazzerini $\mathrm{M}$ et al., 2016. Mortality and its risk factors in Malawian children admitted to hospital with clinical pneumonia, 2001-12: a retrospective observational study. Lancet Glob Health 4: e57-e68.

17. Olson D, Preidis GA, Milazi R, Spinler JK, Lufesi N, Mwansambo C, Hosseinipour MC, McCollum ED, 2013. Task shifting an inpatient triage, assessment and treatment programme improves the quality of care for hospitalised Malawian children. Trop Med Int Health 18: 879-886.

18. King $C$ et al., 2019. Performance of a novel reusable pediatric pulse oximeter probe. Pediatr Pulmonol 54: 1052-1059.

19. Enarson PM, Gie R, Enarson DA, Mwansambo C, 2009. Development and implementation of a national programme for the management of severe and very severe pneumonia in children in Malawi. PLoS Med 6: e1000137.

20. Fleming S, Thompson M, Stevens R, Heneghan C, Plüddemann A, Maconochie I, Tarassenko L, Mant D, 2011. Normal ranges of heart rate and respiratory rate in children from birth to 18 years of age: a systematic review of observational studies. Lancet Lond Engl 377: 1011-1018.

21. White IR, Royston $P$, Wood AM, 2011. Multiple imputation using chained equations: issues and guidance for practice. Stat Med 30: 377-399.

22. Bell ML, Fairclough DL, 2014. Practical and statistical issues in missing data for longitudinal patient-reported outcomes. Stat Methods Med Res 23: 440-459.

23. West JB, 1973. Respiratory Physiology: the Essentials. Baltimore, MD: Williams \& Wilkins.

24. Lee WW, Mayberry K, Crapo R, Jensen RL, 2000. The accuracy of pulse oximetry in the emergency department. Am J Emerg Med 18: 427-431.

25. World Health Organization, 2013. Pocket Book of Hospital Care for Children: Guidelines for the Management of Common Childhood IIInesses, 2nd edition. Geneva, Switzerland: WHO.

26. Subhi R, Adamson M, Campbell H, Weber M, Smith K, Duke T, 2009. The prevalence of hypoxaemia among ill children in developing countries: a systematic review. Lancet Infect Dis 9: 219-227.

27. English $\mathrm{M}$ et al., 2003. Causes and outcome of young infant admissions to a Kenyan district hospital. Arch Dis Child 88: 438-443.

28. Onyango FE, Steinhoff MC, Wafula EM, Wariua S, Musia J, Kitonyi $\mathrm{J}, 1993$. Hypoxaemia in young Kenyan children with acute lower respiratory infection. BMJ 306: 612-615.

29. WHO, 2016. Paediatric Emergency Triage, Assessment and Treatment: care of Critically-III Children. Available at: http:// www.who.int/maternal_child_adolescent/documents/paediatricemergency-triage-update/en/. Accessed November 20, 2017.

30. Florin TA, Ambroggio L, Brokamp C, Rattan MS, Crotty EJ, Kachelmeyer A, Ruddy RM, Shah SS, 2017. Reliability of examination findings in suspected community-acquired pneumonia. Pediatrics 140: e20170310.

31. McCollum ED, Bjornstad E, Preidis GA, Hosseinipour MC, Lufesi N, 2013. Multicenter study of hypoxemia prevalence and quality of oxygen treatment for hospitalized Malawian children. Trans $R$ Soc Trop Med Hyg 107: 285-292. 